\title{
Effect of Linguizhugan decoction on neuroinflammation and expression disorder of the amyloid $\beta$-related transporters RAGE and LRP-1 in a rat model of Alzheimer's disease
}

\author{
QIANFENG HU ${ }^{1 *}$, BEIBEI YU ${ }^{1 *}$, QINLEI CHEN ${ }^{2}$, YI WANG $^{1}$, YUN LING $^{1}$, \\ SONGXIAN SUN ${ }^{1}$, YINLONG SHI ${ }^{1}$ and CHUNXIANG ZHOU ${ }^{1,3}$

\begin{abstract}
${ }^{1}$ Department of Febrile Disease, Basic Medicine College, Nanjing University of Chinese Medicine, Nanjing, Jiangsu 210046;
${ }^{2}$ Department of Infectious Disease, Jiangsu Province Hospital of Traditional Chinese Medicine, Nanjing, Jiangsu 210000;

${ }^{3}$ Department of Traditional Chinese Medicine, Nanjing BenQ Hospital, Nanjing, Jiangsu 210036, P.R. China
\end{abstract}

Received August 18, 2016; Accepted October 2, 2017

DOI: $10.3892 / \mathrm{mmr} .2017 .7983$

\begin{abstract}
Linguizhugan decoction (LGZG), a notable prescription in Traditional Chinese Medicine, is a classical formula for the treatment of Alzheimer's disease (AD), inflammatory injury and fluid retention. The present study aimed to investigate the neuroprotective effect of LGZG on an amyloid $\beta(\mathrm{A} \beta)$-induced AD rat model. Sprague-Dawley rats were administered with $A \beta 1-42$ to induce $A D$ and inflammatory responses, and subsequently with LGZG (4.8, 2.4 or $1.2 \mathrm{~g} / \mathrm{kg}$ ), donepezil ( $2 \mathrm{mg} / \mathrm{kg}$ ) or distilled water for 30 consecutive days. Learning and memory behaviors were evaluated via Morris water maze test. The neuronal impairment of AD rats was observed via hematoxylin-eosin staining. The levels of pro-inflammatory cytokines, and $A \beta$ in the brain tissue were detected with ELISA kits. Protein expression levels of mitogen-activated protein kinase and nuclear factor- $\kappa \mathrm{B}$ signalling were measured by western blot analysis. The expression of lipoprotein receptor-related protein-1 (LRP-1) and receptor for advanced glycation endproducts (RAGE) in the brain were detected by western blot analysis, reverse transcription-quantitative polymerase chain reaction and immunohistochemistry analysis. LGZG was demonstrated to significantly improve learning and memory ability, and ameliorate neuroinflammation in AD rats. LGZG
\end{abstract}

Correspondence to: Professor Chunxiang Zhou, Department of Febrile Disease, Basic Medicine College, Nanjing University of Chinese Medicine, 138 Xianlin Road, Nanjing, Jiangsu 210046, P.R. China

E-mail: chunxiangzhiou@163.com

*Contributed equally

Key words: Alzheimer's disease, Lingguizhugan decoction, receptor for advanced glycation endproducts, lipoprotein receptor-related protein-1, amyloid $\beta$ protein fragment $1-42$, mitogen-activated protein kinase, nuclear factor- $\kappa \mathrm{B}$ increased the levels of LRP-1 and decreased the levels of RAGE. Furthermore, the present results demonstrated that LGZG treatment significantly inhibited MAPK and NF- $\kappa \mathrm{B}$ signalling, and reduced the production of pro-inflammatory cytokines and $A \beta$ accumulation in AD rats. $L G Z G$ exhibited a potential protective effect on $A \beta 1-42$-induced AD by regulating $A \beta$ transportation, and inhibiting RAGE/MAPK and $\mathrm{NF}-\kappa \mathrm{B}$ signalling. These results suggest that LGZG may be considered for the treatment of AD.

\section{Introduction}

Alzheimer's disease (AD) is a neurodegenerative disorder with pathological hallmarks including senile plaques induced by abnormal deposition of amyloid- $\beta(\mathrm{A} \beta)$ and neurofibrillary tangles (NFTs) formed by aberrantly hyperphorylated tau protein. It is well known that $A \beta$ has a critical role in the pathogenesis of $\mathrm{AD}$, as the accumulation of $\mathrm{A} \beta$ leads to neuroinflammation and induces neuronal damage (1). One of the most important mechanisms of $\mathrm{A} \beta$ accumulation in the brain is transshipment imbalances through the blood-brain barrier (BBB) (2), and two primary influx and efflux receptors have been implicated in this process: Receptor for advanced glycation endproducts (RAGE) and lipoprotein receptor-related protein-1 (LRP-1) (3-5). RAGE acts as an important transporter via regulating influx of circulating $A \beta$ into the brain (6), whereas LRP1 mediates the efflux of brain-derived $A \beta$ into the circulation via the BBB (7). Furthermore, RAGE is a multi-ligand receptor of the immunoglobulin superfamily of cell surface molecules, with RAGE ligands consisting of advanced glycation end products and $A \beta$ proteins (8). It has been indicated that these ligand-receptor interactions are able to activate receptor-mediated signaling pathways, including the mitogen-activated protein kinase (MAPK) and nuclear factor (NF) $\kappa$ B pathway (9), and subsequently promote the production of pro-inflammatory cytokines, such as interleukin (IL)-1 $\beta$, IL-6 and tumor necrosis factor (TNF)- $\alpha(10,11)$. Therefore, RAGE/MAPK, the NF- $\kappa \mathrm{B}$ signaling pathway and the downstream inflammatory response may be a potential therapeutic target for $\mathrm{A} \beta$-induced brain damage. 
Lingguizhugan decoction (LGZG) is composed of Poria (the dried fungus nucleus of Poria cocos (Schw.) Wolf), Cinnamomi ramulus (the dried twigs of Cinnamomum cassia (L.) J. Presl), Atractylodis macrocephalae rhizoma (the fried and dried rhizome of Atractylodes macrocephala Koidz) and Glycyrrhizae radix et rhizoma (the dried roots and rhizome of Glycyrrhizae uralensis Fisch.), and is widely used in Traditional Chinese Medicine. The formula originates from Treatise on Febrile Diseases (12), and has been used for the treatment of $\mathrm{AD}$, inflammatory injury and fluid retention (13). It has previously been demonstrated that LGZG inhibits the levels of pro-inflammatory cytokines produced by $\mathrm{A} \beta_{1-42}$ induced BV-2 microglia cells (14). These findings indicate the potential of LGZG in the treatment of AD; however, the molecular mechanism remains unclear. Therefore, the aim of the present study was to investigate the effect of LGZG on $\mathrm{A} \beta$-induced $\mathrm{AD}$ rats.

\section{Materials and methods}

Animals. All experiments and animal care protocols in the present study were performed in accordance with the Guide for the Care and Use of Laboratory Animals (15). Ethical approval for these protocols was granted by the Medical Ethics Committee of Nanjing University of Traditional Chinese Medicine [Nanjing, China; approval no. ACU-06(20151113)].

Male Sprague-Dawley rats $(\mathrm{n}=56 ; 8$ week-old, weight, 200-250 g) were purchased from Shanghai SLAC Laboratory Animal Co., Ltd. (Shanghai, China) and allowed 1 week to adapt to the laboratory environment prior to experiments. Rats were housed at $50 \pm 10 \%$ humidity, $24 \pm 2^{\circ} \mathrm{C}$ and a 12 -h light/dark cycle with ad libitum access to standard chow and water.

Rats were randomly divided into seven experimental groups: Control group, sham group (saline + sterile distilled water), model group (A $\beta 1-42+$ sterile distilled water), $L G Z G$ 4.8 group $(\mathrm{A} \beta 1-42+4.8 \mathrm{~g} / \mathrm{kg}$ LGZG), LGZG 2.4 group $(\mathrm{A} \beta 1-42+2.4 \mathrm{~g} / \mathrm{kg} \mathrm{LGZG)}$, LGZG 1.2 group $(\mathrm{A} \beta 1-42+$ $1.2 \mathrm{~g} / \mathrm{kg} \mathrm{LGZG}$ ) and positive control group [A $\beta 1-42+2 \mathrm{mg} / \mathrm{kg}$ donepezil hydrochloride (DNP)]. Each group contained 8 rats.

Reagents and administration procedures. Poria (Anhui, China), Cinnamomi ramulus (Guangdong, China), Atractylodis macrocephalae rhizoma (Jiangsu, China), and Glycyrrhizae radix et rhizoma (Xinjiang, China) were purchased from Jiangsu Lianshui Pharmaceutical Co., Ltd. (Lianshui, China), and prepared in a dry weight ratio of 4:3:2:2 for the production of LGZG. The constituents were then mixed with $\mathrm{x} 10$ the quantity of distilled water, decocted for $2 \mathrm{~h}$ and again for $1 \mathrm{~h}$, pressure-filtered, concentrated using a rotating evaporator to obtain a $1 \mathrm{~g} / \mathrm{ml}$ solution and stored at $4{ }^{\circ} \mathrm{C}$ prior to further use. $\mathrm{A} \beta 1-42$ (cat no. 107761-42-2) and monoclonal mouse GAPDH (cat no. 9001-50-7) antibodies were obtained from Sigma-Aldrich (Merck KGaA, Darmstadt, Germany). Antibodies against LRP-1 (cat no. 64099), RAGE (cat no. 4679), phosphorylated (p)-NF- $\mathrm{kB}$ (cat no. 3033), NF-kB

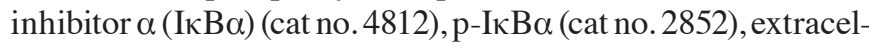
lular signal-regulated kinase (Erk)1/2 (cat no. 9102), p-Erk1/2 (cat no. 4370), p38 (cat no. 9212) and p-p38 (cat no. 9211), as well as horseradish peroxidase (HRP)-conjugated anti-mouse (cat no. 7072) or anti-rabbit (cat no. 7071) IgG secondary antibodies were obtained from Cell Signaling Technology, Inc. (Danvers, MA, USA). Rabbit anti-rat antibodies specific against LRP-1 and RAGE were obtained from Beyotime Institute of Biotechnology, Haimen, China (cat no. A0208). ELISA kits for TNF- $\alpha$ (cat no. JEB-13718), IL-1 $\beta$ (cat no. JEB-13503), IL-6 (cat no. JEB-13729) and A $\beta$ (cat no. JEB-15109) were purchased from Nanjing KJI Biological Technology Development Co., Ltd. (Nanjing, China). Other chemicals were purchased from Merck KGaA.

Following anesthesia with intraperitoneal injection of $0.45 \%$ pentobarbital sodium $(40 \mathrm{mg} / \mathrm{kg})$, rats were secured on a stereotaxic device. Either peptides (A $\beta 1-42 ; 10 \mu \mathrm{g}$; Sigma-Aldrich, Merck KGaA) or vehicle (saline) was administered into the bilateral hippocampus CA1, according to the rat brain in stereotaxic coordinates $(-3.0 \mathrm{~mm}$ posterior to bregma, $\pm 2.0 \mathrm{~mm}$ lateral to midline, and $-3.0 \mathrm{~mm}$ dorsal to ventral dura). These were supplied progressively within a 5 -min duration, followed by a cessation period of $5 \mathrm{~min}$, as described previously (16-18). Rats were subsequently administered with 200,000 U/ml penicillin-G once daily for 3 days following surgery. Following the procedure, LGZG (1.2, 2.4 and $4.8 \mathrm{~g} / \mathrm{kg}$ ), $2 \mathrm{mg} / \mathrm{kg}$ DNP and vehicle (sterile distilled water) were administered orally to the rats twice daily by gavage for 30 days.

Morris water maze test. The water-maze test was performed 25 days following drug administration. This test was performed in a circular pool $(180 \mathrm{~cm}$ diameter $x 60 \mathrm{~cm}$ height). A hidden circular platform was submerged $2 \mathrm{~cm}$ below the water level in one pool quadrant. Rats were allowed two trial sessions each day for 4 successive days with an intermission of $5 \mathrm{~h}$ between trials. A video tracking system was used to view the complete procedure. When the rat had located the platform successfully, it was permitted to remain there for $10 \mathrm{sec}$. Rats was physically positioned on the platform for $10 \mathrm{sec}$ in cases where they did not succeed in locating the platform within $90 \mathrm{sec}$, wherein escape latency was marked as $120 \mathrm{sec}$. A single probe trial was performed on the fifth day.

Hematoxylin-eosin $(H \& E)$ staining. Rats were anesthetized with $0.45 \%$ pentobarbital sodium, then sacrificed via perfusion transcardially with $0.1 \mathrm{~mol} / 1 \mathrm{PBS}(\mathrm{pH} 7.4$ ) and $4 \%$ paraformaldehyde. Sections of the hippocampi were harvested and fixed in $4 \%$ formalin at $4^{\circ} \mathrm{C}$ for $\geq 72 \mathrm{~h}$, dehydrated in graded alcohol series, and embedded in paraffin. Specimens were cut in 5- $\mu \mathrm{m}$ sections on a rotary microtome and mounted on 3-aminopropyltriethoxysilane-coated glass slides, then stained at $4^{\circ} \mathrm{C}$ for 20 min with $\mathrm{H} \& \mathrm{E}$ and light microscopic examination for histopathological alteration was performed (Olympus BH2; Olympus Corporation, Tokyo, Japan) as described previously $(19,20)$. Images were captured using a Nikon Coolpix 990 camera (Nikon Corporation, Tokyo, Japan) with an original magnification of $\times 200$.

Immunohistochemistry of LRP-1 and RAGE. For immunohistochemical analysis, brain tissue was fixed in $4 \%$ paraformaldehyde at $4^{\circ} \mathrm{C}$ for $24 \mathrm{~h}$, embedded in paraffin, and then cut into $4 \mu \mathrm{m}$-sections, hydrated and incubated in $3 \%$ hydrogen peroxide at room temperature for $10 \mathrm{~min}$. The 
sections were washed three times with PBS and then stored at $4^{\circ} \mathrm{C}$ in PBS supplemented with $3 \%$ bovine serum albumin (cat no. B2064, Sigma-Aldrich, Merck KGaA) for $30 \mathrm{~min}$. Sections were then incubated with antibodies against LRP-1 (1:200) or RAGE (1:200) overnight at $4^{\circ} \mathrm{C}$, and a negative control was incubated with PBS. These sections were then exposed to biotinylated universal secondary antibodies (1:5,000; cat no. A0208; Beyotime Institute of Biotechnology) for $1 \mathrm{~h}$ and subsequently to streptavidin biotin horseradish peroxidase solution $(1: 1,000)$ at room temperature for $1 \mathrm{~h}$, following washing with PBS. And subsequently stained with 3,3'-diaminobenzidine solution for $45 \mathrm{sec}$ at $37^{\circ} \mathrm{C}$. Sections were counterstained with hematoxylin at room temperature for $20 \mathrm{sec}$. Graded alcohols were then used to dehydrate dehydrate section, and they were subsequently fixed in neutral balsam (Sinopharm Chemical Reagent Co., Ltd., Shanghai, China) at $60^{\circ} \mathrm{C}$ for $30 \mathrm{~min}$. LRP-1 and RAGE staining was evaluated using Image-Pro Plus 6.0 software (Media Cybernetics, Inc., Rockville, MD, USA).

ELISA of TNF- $\alpha, I L-1 \beta, I L-6$, and $A \beta$. Rats were decapitated 60 min after the behavioral tests. Brains were immediately harvested, weighed and homogenized using $0.9 \%$ ice-cold saline water. The homogenate was centrifuged at $3,000 \mathrm{x} \mathrm{g}$ for $10 \mathrm{~min}$ at $4^{\circ} \mathrm{C}$ and the supernatants were harvested and served at $4^{\circ} \mathrm{C}$ prior to ELISA analysis. The expressions of TNF- $\alpha$, IL-1 $\beta$, IL- 6 , and A $\beta$ in brain tissues of rats were measured using ELISA kits according to the manufacturer's instructions.

Western blot analysis. To determine the expression levels of LRP-1, RAGE, p-NF- $\kappa$ B, I $\mathrm{B} \alpha, \mathrm{p}-\mathrm{I} \kappa \mathrm{B} \alpha$, Erk1/2, p-Erk1/2, p38, p-p38 in the brain, western blotting was performed as described previously (20). The rat brain tissues were homogenized (centrifuged at $14,000 \times \mathrm{g}, 4^{\circ} \mathrm{C}, 15 \mathrm{~min}$ ), washed with PBS, and incubated in lysis buffer (cat no. P0013; Beyotime Institute of Biotechnology) containing protease inhibitor cocktail (cat no. P8330, Sigma-Aldrich; Merck KGaA) to isolate brain protein samples. A total of $30 \mu \mathrm{g}$ protein samples were separated by $10 \%$ SDS-PAGE gels and blotted onto nitrocellulose membranes with TBST buffer $(10 \mathrm{mM}$ Tris- $\mathrm{HCl}, 150 \mathrm{mM} \mathrm{NaCl}, 0.1 \%$ v/v Tween-20) containing 5\% nonfat milk for $1 \mathrm{~h}$ at room temperature. Membranes were incubated with primary antibodies against LRP-1, RAGE, p-NF- $\kappa \mathrm{B}, \mathrm{I} \kappa \mathrm{B} \alpha, \mathrm{p}-\mathrm{I} \kappa \mathrm{B} \alpha$, Erk1/2, p-Erk1/2, p38, and p-p38 $(1: 1,000)$ at $4^{\circ} \mathrm{C}$ overnight, with GAPDH $(1: 1,000)$ was used as loading control. Membranes were subsequently washed three times in $0.05 \%$ Tween 20 in TBS for 5 min and then incubated with HRP-conjugated secondary antibodies $(1: 5,000)$ for $1 \mathrm{~h}$ at room temperature. Enhanced chemiluminescence (cat no. NEL103E001EA; PerkinElmer, Inc., Waltham, MA, USA) was used to detect the signals. Quantity One software version 4.62 (Bio-Rad Laboratories, Inc., Hercules, CA, USA) was used for densitometry analysis.

Reverse transcription-quantitative polymerase chain reaction $(R T-q P C R)$ analysis. Total RNA was extracted using TRIzol reagent (cat no. 15596026; Thermo Fisher Scientific, Inc.) according to the manufacturer's protocol and reverse transcribed to produce cDNA using a TaqMan reverse transcription reagent kit (Thermo Fisher Scientific, Inc.). cDNA was then amplified via PCR with the following primers: RAGE, forward 5'-GACCACTCCTACCTATTC CT-3' and reverse 5'-TCCACCTTCAGGCTCAACCA-3'; LRP-1, forward 5'-CGTCACTTACATCAACAACC-3', and reverse 5'-CAGCCATTCACATTTCTTGC-3'; and GAPDH, forward 5'-GAGCTGAACGGGAAACTCAC-3', and reverse 5'-GGTCTGGGATGGAAACTGTG-3'. The thermal cycling conditions included $5 \mathrm{~min}$ at $94^{\circ} \mathrm{C}$ followed by 32 cycles of $30 \mathrm{sec}$ at $94^{\circ} \mathrm{C}, 45 \mathrm{sec}$ at $55^{\circ} \mathrm{C}$, and $45 \mathrm{sec}$ at $72^{\circ} \mathrm{C}$. The abundance of the mRNA expression level was measured according to the $2^{-\Delta \Delta \mathrm{Cq}}$ method as described previously (21).

Statistical analysis. Data are expressed as the mean + standard deviation. Statistical analysis was performed using the SPSS 19.0 (IBM Corp., Armonk, NY, USA). Statistical differences between groups were determined using one-way analysis of variance with Bonferroni's multiple comparison test using software EZR version 3.1.2 (Jichi Medical University, Tochigi, Japan; www.jichi.ac.jp/saitama-sct/SaitamaHP.files/statmedEN.html). $\mathrm{P}<0.05$ was considered to indicate a statistically significant difference.

\section{Results}

$L G Z G$ ameliorates memory deficits of AD rats. As presented in Fig. 1A, the mean escape latency in the Morris water maze declined progressively in all groups throughout the training period. No significant difference was observed between the control and sham-operated groups. A $\beta$-treated rats spent significantly more retentive time on reaching the platform vs. controls $(\mathrm{P}<0.01)$, which indicated notable cognitive damage in $A \beta$-treated rats. Furthermore, this increase in escape latency was significantly ameliorated by LGZG $(1.2 \mathrm{~g} / \mathrm{kg}$ : day $3, \mathrm{P}<0.05 ; 2.4 \mathrm{~g} / \mathrm{kg}$ : day 2 and $4, \mathrm{P}<0.05 ; 4.8 \mathrm{~g} / \mathrm{kg}$ : day $1, \mathrm{P}<0.05$, day $2-4, \mathrm{P}<0.01$ vs. the model) and donepezil (day 1, $\mathrm{P}<0.05$; day $2-4, \mathrm{P}<0.01$ vs. the model) treatment. The swim routes followed by the rats during the second trial on day 2 and day 4 are illustrated in Fig. 1B. The rats seemed to show the tendency to enter all four quadrants of the pool on day 2. On day 4, the control rats were observed to swim in the direction of the platform, whereas $A \beta$-treated rats took lengthier swimming routes. In the probe test, as shown in Fig. 1C and D, the control and sham operated rats spent more time in the target quadrant $(26.88 \pm 5.41$ and $26.75 \pm 5.55 \mathrm{sec}$, respectively) and exhibited greater crossing times $(6.13 \pm 1.25$ and $5.25 \pm 1.03 \mathrm{sec}$, respectively) than the model group rats $(15.13 \pm 3.04 \mathrm{sec}, \mathrm{P}<0.01$ vs. control; $1.88 \pm 0.83 \mathrm{sec}, \mathrm{P}<0.01$ vs. control). When compared with the model group rats, LGZG (4.8 and $2.4 \mathrm{~g} / \mathrm{kg}$ ) and donepezil treated rats exhibited increased crossing times $(\mathrm{P}<0.01, \mathrm{P}<0.05$ and $\mathrm{P}<0.01$, respectively). As for time in the target quadrant, a noteworthy enhancement was observed in the LGZG group at a dose of $2.4(\mathrm{P}<0.05)$ and $4.8 \mathrm{~g} / \mathrm{kg}(\mathrm{P}<0.01)$ as well as the donepezil group $(\mathrm{P}<0.01)$. This data suggests that $\mathrm{LGZG}$ is able to improve the memory and learning ability of the A $\beta$-induced model rats in the Morris water maze test.

LGZG improves brain neuronal damage. In the present study, no neuronal damage was observed in the rats in the control 

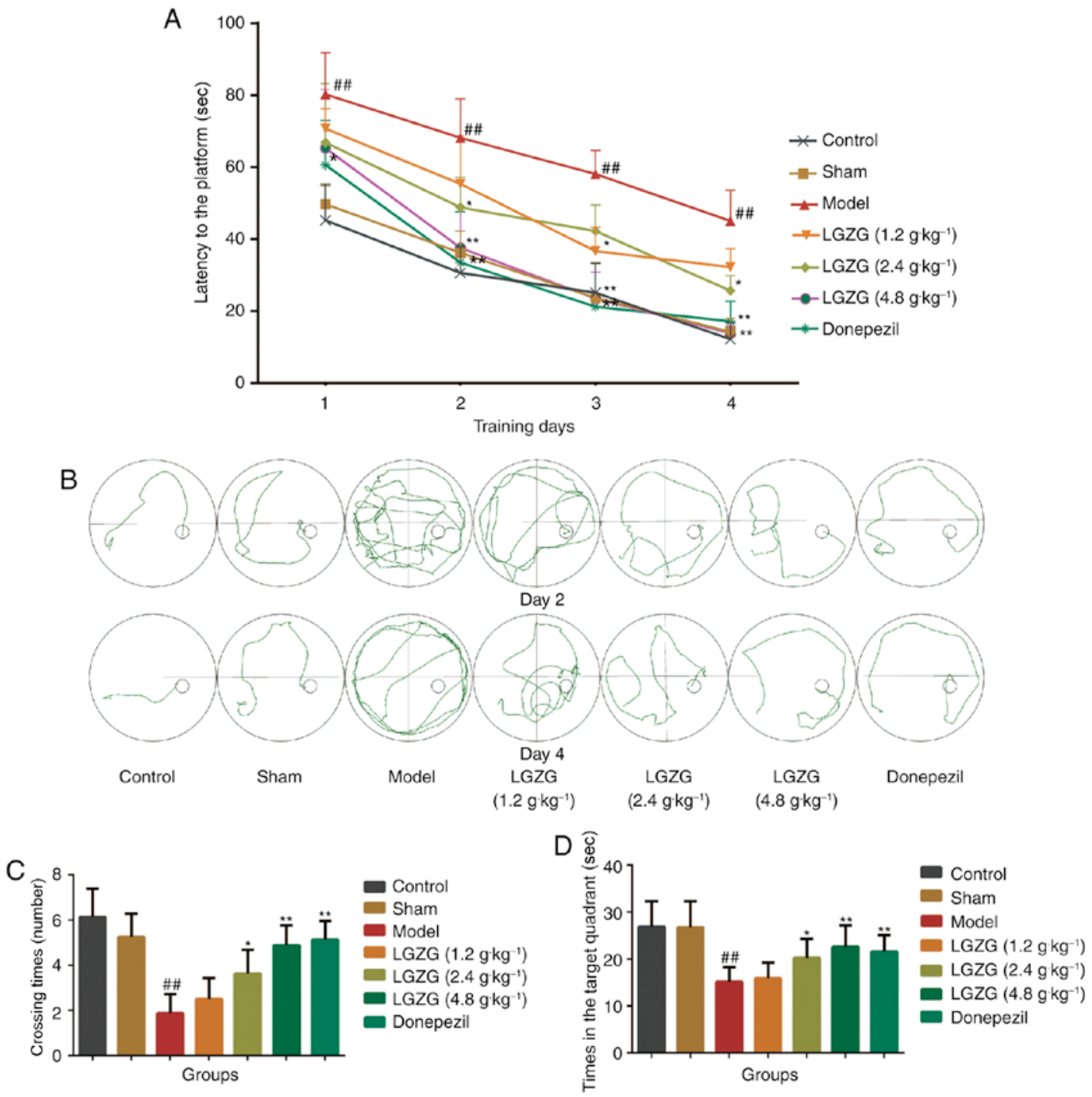

Figure 1. Effects of LGZG on the spatial learning and memory ability of rats in all groups in the Morris water maze test. (A) Latency to mount the escape platform in the water maze during the 4 training days. (B) Representative tracks of the probe trial of rats in the second trial on days 2 and 4 . Traces show the swim path of all groups of rats. (C) Times of crossing the target platform within $120 \mathrm{sec}$. (D) Time spent searching for the target quadrant within 120 sec. Data are presented as the mean + standard deviation ( $\mathrm{n}=8$ per group). ${ }^{\# \#} \mathrm{P}<0.01$ vs. control; ${ }^{*} \mathrm{P}<0.05,{ }^{* * *} \mathrm{P}<0.01$ vs. model. LGZG, Lingguizhugan decoction.

and sham groups. However, rats in the model group exhibited marked neuronal loss in the hippocampus. HE staining also indicated that the number of neurons in the LGZG $(4.8 \mathrm{~g} / \mathrm{kg})$ and donepezil rats was markedly increased vs. the model (Fig. 2). These results indicate that LGZG has a neuroprotective effect on the nerve cells of the brain in $\mathrm{AD}$ rats.

Effects of $L G Z G$ on levels of TNF- $\alpha, I L-1 \beta, I L-6$, and $A \beta 1-42$ in the brain. The results of ELISA analysis (Fig. 3) demonstrate the anti-inflammatory and neuroprotective effects of LGZG in rats with AD. There was a significant increase in the levels of TNF- $\alpha$, IL-1 $\beta$, IL- 6 , and A $\beta 1-42$ in the model group $(\mathrm{P}<0.01$ vs. control). LGZG at doses of $4.8(\mathrm{P}<0.01)$ and $2.4 \mathrm{~g} / \mathrm{kg}(\mathrm{P}<0.05)$, as well as donepezil $(\mathrm{P}<0.01)$, significantly decreased the levels of TNF- $\alpha$, IL-1 $\beta$, IL- 6 , and A $\beta 1-42$ compared with the model. There were significant differences in the levels of IL-6 between the group of LGZG at a dose of $1.2 \mathrm{~g} / \mathrm{kg}(\mathrm{P}<0.05)$ and the model group. Notably, LGZG at $1.2 \mathrm{~g} / \mathrm{kg}$ demonstrated a tendency to decrease pro-inflammatory cytokines levels
(TNF- $\alpha$ and IL-1 $\beta$ ) levels, but no significant difference was observed. These findings suggest that LGZG is able to inhibit neuroinflammation and decrease $A \beta 1-42$ levels in the brain of $\mathrm{AD}$ rats.

LGZG modulates the expression of MAPK and the NF- $\kappa B$ pathway in the brain. To evaluate the expression of MAPK and the NF- $\kappa \mathrm{B}$ pathway, the protein levels in the brain of the rats were analyzed using western blot analysis. As presented in Fig. 4, A $\beta 1-42$ administration significantly increased the protein levels of p-Erk, p-p38, p-NF- $\mathrm{BB}$ and $\mathrm{p}-\mathrm{I} \kappa \mathrm{B} \alpha(\mathrm{P}<0.01$ vs. control) in rats, which indicated activation of MAPK and the NF- $\kappa B$ pathway in AD rats. However, LGZG treatment significantly ameliorated the expression levels of $\mathrm{p}-$ Erk $1 / 2$, and p-p38 at 2.4 and $4.8 \mathrm{~g} / \mathrm{kg}(\mathrm{P}<0.01)$ in the brain of $\mathrm{AD}$ rats, compared with the model. Furthermore, the protein levels of p-NF- $\kappa \mathrm{B}$ and $\mathrm{p}-\mathrm{I} \kappa \mathrm{B} \alpha$ at 2.4 (p-NF- $\kappa \mathrm{B}, \mathrm{P}<0.05 ; \mathrm{p}-\mathrm{I} \kappa \mathrm{B} \alpha$, $\mathrm{P}<0.01)$ and $4.8 \mathrm{~g} / \mathrm{kg}(\mathrm{P}<0.01)$ LGZG were also significantly downregulated compared with the model. These results 


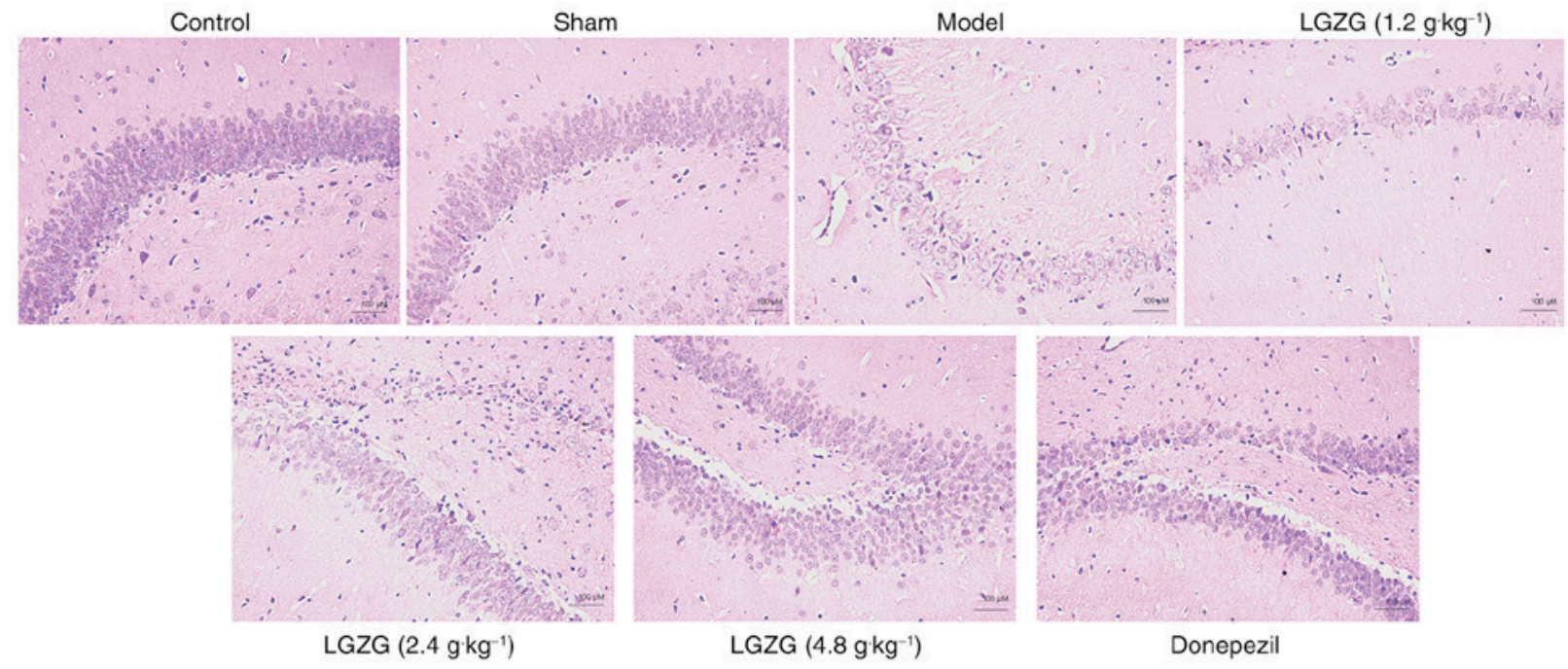

Figure 2. Effects of LGZG on the morphology and number of neurons in an amyloid $\beta$-induced Alzheimer's disease rat model. Light micrographs of hippocampal neurons from the CA1 region of rats orally administered sterile distilled water (control, sham and model), $\mathrm{LGZG}$ at doses of 4.8, 2.4 and 1.2 g/kg, or $2 \mathrm{mg} / \mathrm{kg}$ donepezil hydrochloride. $\mathrm{n}=8$ per group. LGZG, Lingguizhugan decoction.

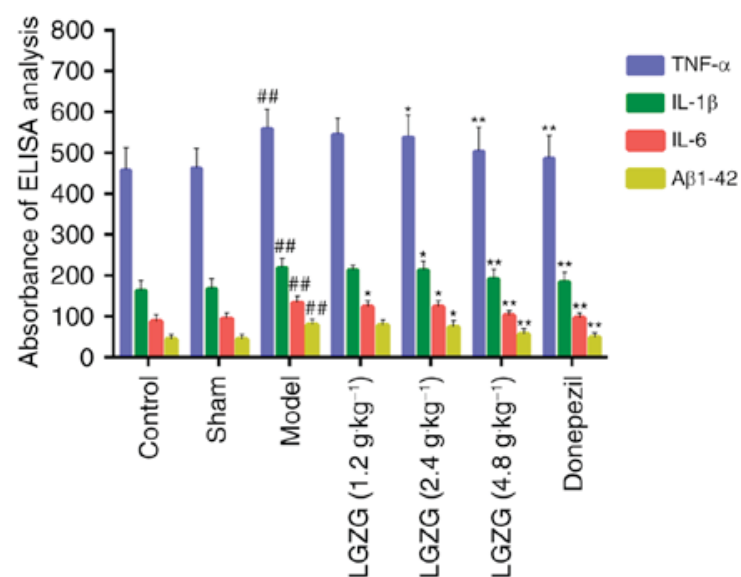

Figure 3. Effects of donepezil and LGZG on the expressions of TNF- $\alpha$, IL-1 $\beta$, IL-6 and A $\beta 1-42$ in hippocampus tissues of Alzheimer's disease-model rats. Data are presented as the mean + standard deviation ( $\mathrm{n}=8$ per group). ${ }^{\# \#} \mathrm{P}<0.01$ vs. control; ${ }^{*} \mathrm{P}<0.05,{ }^{* *} \mathrm{P}<0.01$ vs. model. LGZG, Lingguizhugan decoction; TNF, tumor necrosis factor; IL, interleukin; $A \beta$, amyloid $\beta$.

suggested that LGZG is able to inhibit MAPK and NF- $\mathrm{B}$ signaling in the brain of $\mathrm{AD}$ rats.

Effect of LGZG on the expression levels of RAGE and LRP-1 in the brain. RAGE and LRP-1 are essential in controlling A $\beta$ balance in the brain (21). In the present study, the expression of RAGE and LRP-1 in the brain of the rats were analyzed by western blot analysis, RT-qPCR and immunohistochemistry. Western blot analysis (Fig. 5A) and RT-qPCR (Fig. 5B) indicated an increased expression of LRP-1, and a decreased expression of RAGE in the brains of LGZG rats compared with model rats. As presented in Fig. 6, the mean densities of LRP-1 were lower (Fig. 6A and C), whereas the mean densities of RAGE were significantly higher (Fig. 6B and D) in the brain of model rats compared with controls. When compared with the model group, LGZG significantly downregulated the reactive expression levels of RAGE and upregulated the reactive expression levels of LRP-1 $(2.4 \mathrm{~g} / \mathrm{kg}, \mathrm{P}<0.05$; $4.8 \mathrm{~g} / \mathrm{kg}, \mathrm{P}<0.01)$.

\section{Discussion}

$\mathrm{AD}$ is one of the most profound neurodegenerative disease in elderly people, which affects cognition, behavior and function (22). In the present study, $A \beta$ administration significantly decreased the ability of learning and memory and induced neuropathological changes in rats, which is in accordance with the results of the previous study (23). Accordingly, it was demonstrated in the present study that LGZG treatment significantly ameliorated learning and memory and $\mathrm{A} \beta$-induced pathologic changes in rats. These results above suggested that the administration of LGZG exhibits neuroprotective effects in AD.

It was recently verified that $A \beta$ accumulation has a key role in the mechanism of neuron damage, and learning and memory function in AD (24). A $\beta$ levels increase significantly in rats following $A \beta$ injection, which indicated the accumulation of $A \beta$ in this model. One explanation is the enhanced circulation in brain transportation and the impaired brain to-blood transportation of $\mathrm{A} \beta$ across the $\mathrm{BBB}$, which have been observed in AD patients and rat models $(25,26)$. In accordance with these reports, the present data demonstrated that RAGE, which is a primary transporter of $A \beta$ across the $\mathrm{BBB}$ into the brain, was upregulated following $\mathrm{A} \beta$ administration. Conversely, LRP-1, which is the transporter of $A \beta$ out of the brain, was downregulated in AD rats. Furthermore, these changes were ameliorated by treatment with LGZG. Therefore, these results indicated that LGZG treatment decreased the accumulation of $A \beta$ in $A D$ rats, possibly by regulating the transport receptors of $A \beta$.

In addition, RAGE ligation by $A \beta$ is able to activate multiple signaling pathways, including MAPK and the NF- $\mathrm{B}$ pathway (9). In the present study, it was reported that AD rats exhibited significantly higher phosphorylation levels of MAPK 

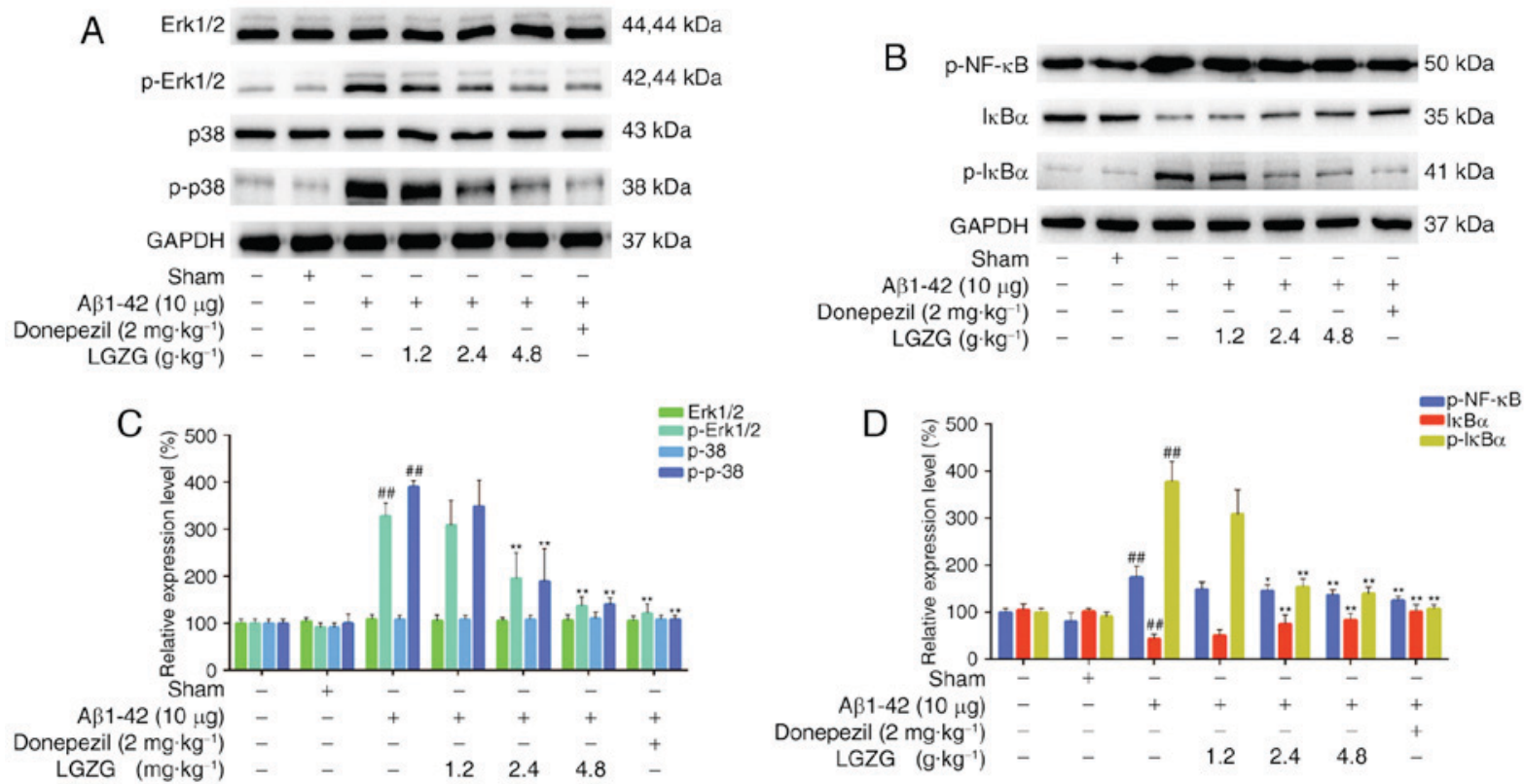

Figure 4. Effects of LGZG on the expression of MAPK and NF-кB pathway in Alzheimer's disease-model rats. (A and B) Representative western blot bands and (C and D) quantification of (A and C) MAPK and (B and D) the NF- $\mathrm{kB}$ pathway. Data are presented as the mean + standard deviation ( $\mathrm{n}=8$ per group). ${ }^{\# \#} \mathrm{P}<0.01$ vs. control; ${ }^{*} \mathrm{P}<0.05,{ }^{* *} \mathrm{P}<0.01$ vs. model. LGZG, Lingguizhugan decoction; MAPK, mitogen-activated protein kinase; NF, nuclear factor; Erk, extracellular signal-regulated kinase; $\mathrm{p}$, phosphorylated; $\mathrm{A} \beta$, amyloid $\beta$; IкB $\alpha, \mathrm{NF}-\kappa \mathrm{B}$ inhibitor $\alpha$.
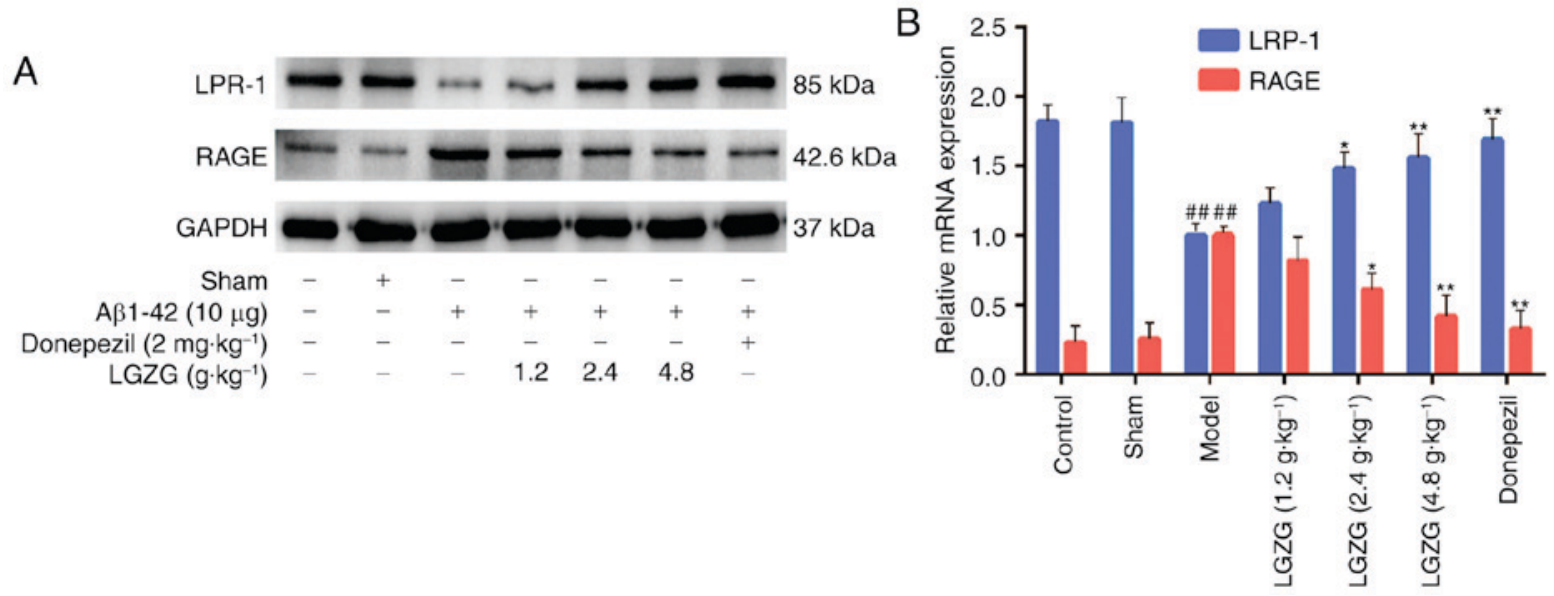

Figure 5. Effect of LGZG on the protein and mRNA levels of LRP-1 and RAGE in Alzheimer's disease-model rats. The expression of RAGE and LRP-1 in rat brains were analyzed by (A) western blotting and (B) reverse transcription-quantitative polymerase chain reaction. Data are presented as the mean + standard deviation ( $\mathrm{n}=8$ per group). ${ }^{\# \#} \mathrm{P}<0.01$ vs. control; ${ }^{*} \mathrm{P}<0.05,{ }^{* *} \mathrm{P}<0.01$ vs. model. LGZG, Lingguizhugan decoction; LRP-1, lipoprotein receptor-related protein-1; RAGE, receptor for advanced glycation endproducts; $A \beta$, amyloid $\beta$.

and NF- $\mathrm{kB}$, which is consistent with previous studies (10). Subsequently, marked increases of pro-inflammatory cytokines were observed in this animal model. However, previous in vitro study have revealed the anti-inflammatory effect of LGZG (-14). In accordance with these studies, the present results demonstrated that LGZG significantly inhibited the activation of MAPK and NF- $\kappa B$, and reduced the $A \beta$-induced elevated levels of TNF- $\alpha$, IL- $1 \beta$ and IL- 6 in AD rats, which suggested that the anti-inflammatory effect may contribute to the protective effects of LGZG in AD rats. These findings indicated that LGZG attenuates $A \beta$-induced AD partially via suppressing the activation of RAGE/MAPK and the NF- $\kappa$ B pathway.

Donepezil is an acetylcholinesterase/cholinesterase inhibitor, which is considered as the standard treatment of AD (27).
Donepezil has been demonstrated to significantly ameliorate memory-associated behavioral deficits and decrease $A \beta$ production (28). In the present study, it was demonstrated that the protective effects of LGZG on learning and memory deficits, as well as neuronal impairment were comparable to those of donepezil. Previous studies have reported that donepezil has cholinergic side effects (29) and induces sleep disturbances (30) in patients with $\mathrm{AD}$. Notably, no obvious side effects or toxic reactions were observed in the groups treated with LGZG. Therefore, the present study suggests that LGZG may be considered for treatment of AD.

In summary, the present study demonstrated the protective effect of LGZG in AD rats. LGZG promoted the ability of learning and memory, and reduced neuron damage and 
A
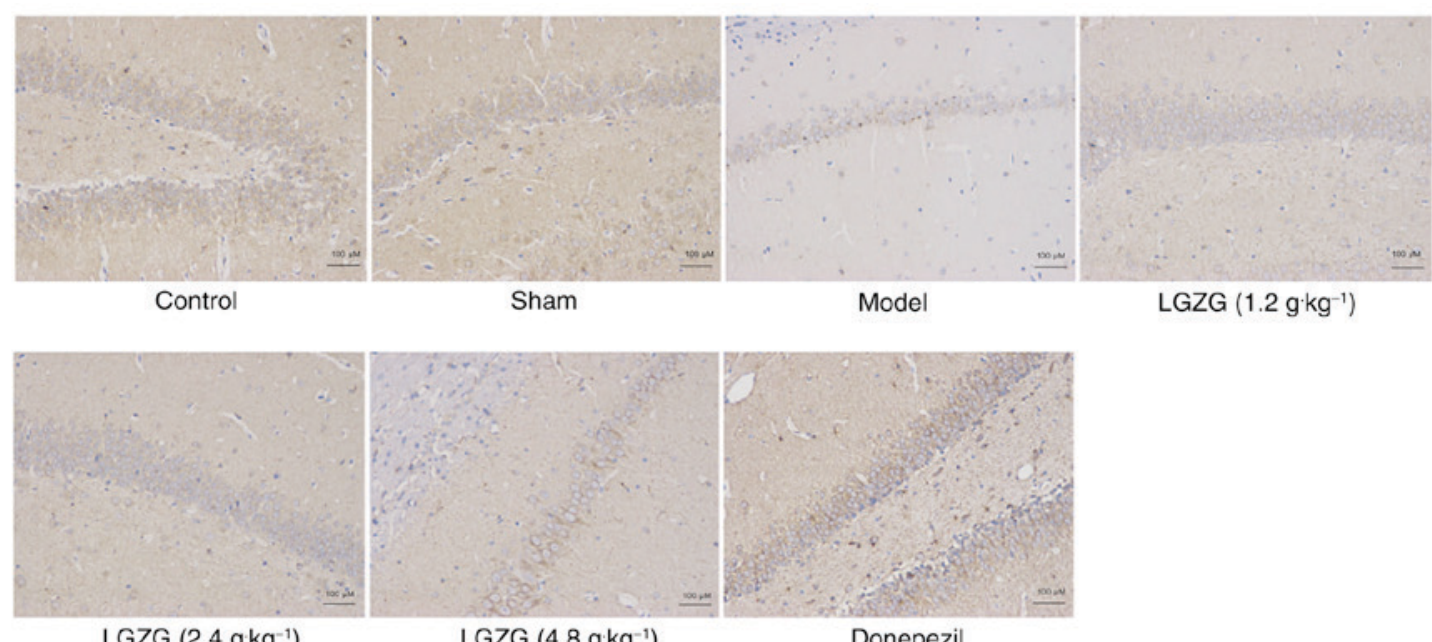

LGZG (2.4 $\left.\mathrm{g} \mathrm{kg}^{-1}\right)$

LGZG (4.8 $\left.\mathrm{g} \mathrm{kg}^{-1}\right)$

Donepezil

B

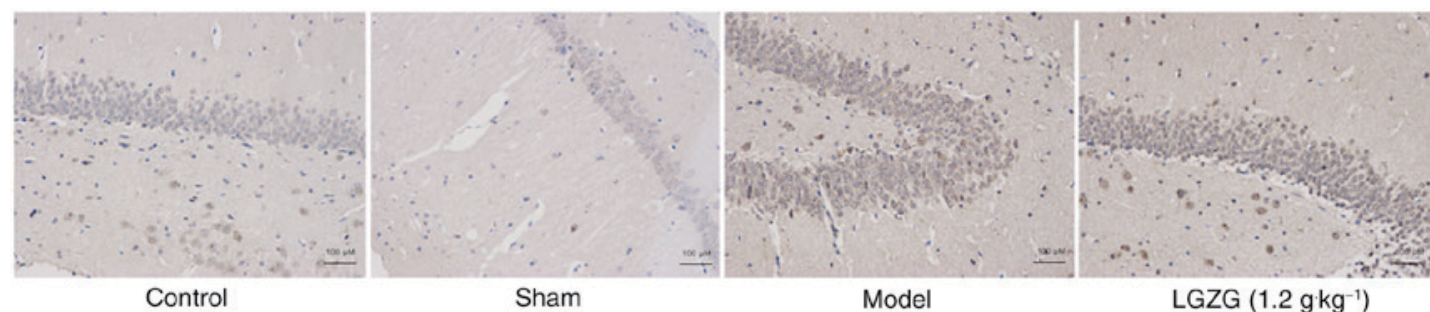

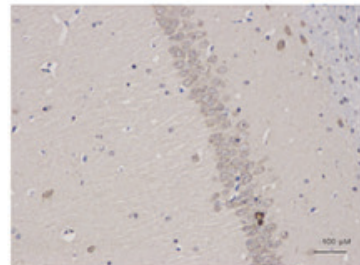

LGZG $\left(2.4 \mathrm{gkg}^{-1}\right)$

C

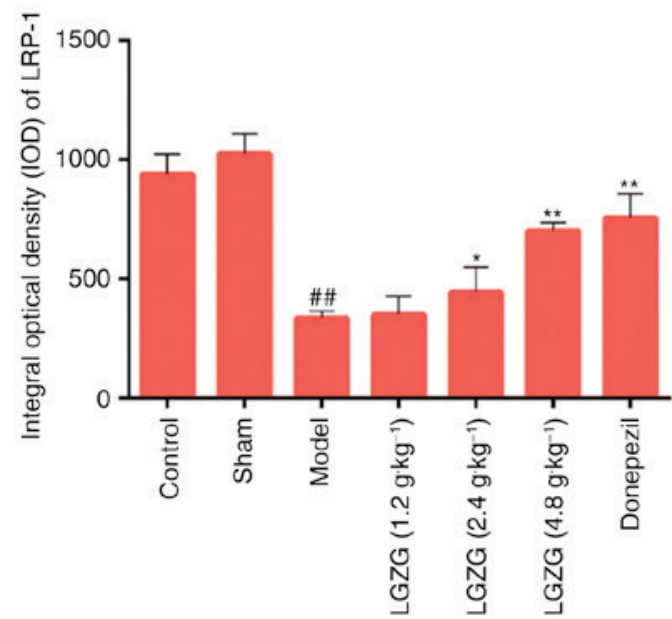

LGZG (4.8 $\left.\mathrm{gkg}^{-1}\right)$

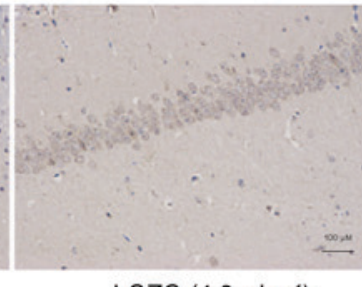

D

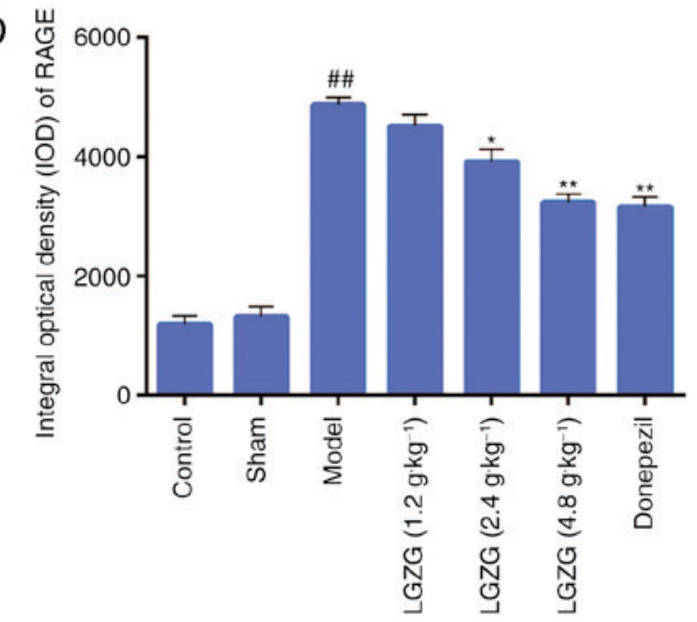

Figure 6. Effect of LGZG on the expression of LRP-1 and RAGE in the brain of Alzheimer's disease-model rats. The expression of (A) RAGE and (B) LRP-1 in the brain were detected by immunohistochemistry. Quantitative analysis of the expression of (C) LRP-1 and (D) RAGE. Data are presented as the mean + standard deviation ( $\mathrm{n}=8$ per group). ${ }^{\# \#} \mathrm{P}<0.01$ vs. control; ${ }^{*} \mathrm{P}<0.05,{ }^{* *} \mathrm{P}<0.01$ vs. model. LGZG, Lingguizhugan decoction; LRP-1, lipoprotein receptor-related protein-1; RAGE, receptor for advanced glycation endproducts; OD, optical density.

inflammation in an $\mathrm{A} \beta$-induced $\mathrm{AD}$ model in rats. The mechanism may be associated with the regulation of $A \beta$ transportation, and inhibition of RAGE/MAPK and NF- $\mathrm{kB}$ signaling by LGZG. Therefore, the present study elucidates the potential pharmacological application of LGZG for the treatment of $\mathrm{AD}$.

\section{Acknowledgements}

The present study was financially supported by the National Natural Science Foundation of China (grant no. 81503485) and the Natural Science Foundation of Jiangsu Province (grant no. BK20161047). 


\section{References}

1. Ezra A, Rabinovich-Nikitin I, Rabinovich-Toidman P and Solomon B: Multifunctional effect of human serum albumin reduces Alzheimer's disease related pathologies in the $3 \times \mathrm{Tg}$ mouse model. J Alzheimers Dis 50: 175-188, 2016.

2. Keaney J, Walsh DM, O'Malley T, Hudson N, Crosbie DE, Loftus T, Sheehan F, McDaid J, Humphries MM, Callanan JJ, et al: Autoregulated paracellular clearance of amyloid- $\beta$ across the blood-brain barrier. Sci Adv 1: e1500472, 2015.

3. Xi YD, Li XY, Ding J, Yu HL, Ma WW, Yuan LH, Wu J and Xiao R: Soy isoflavone alleviates A $\beta 1-42$-induced impairment of learning and memory ability through the regulation of RAGE/LRP-1 in neuronal and vascular tissue. Curr Neurovasc Res 10: 144-156, 2013.

4. Do TM, Dodacki A, Alata W, Calon F, Nicolic S, Scherrmann JM, Farinotti R and Bourasset F: Age-dependent regulation of the Blood-brain barrier Influx/Efflux equilibrium of amyloid- $\beta$ peptide in a mouse model of Alzheimer's disease (3xTg-AD). J Alzheimers Dis 49: 287-300, 2016.

5. Matsumoto K, Chiba Y, Fujihara R, Kubo H, Sakamoto H and Ueno M: Immunohistochemical analysis of transporters related to clearance of amyloid- $\beta$ peptides through blood-cerebrospinal fluid barrier in human brain. Histochem Cell Biol 144: 597-611, 2015.

6. Deane R, Singh I, Sagare AP, Bell RD, Ross NT, LaRue B Love R, Perry S, Paquette N, Deane RJ, et al: A multimodal RAGE-specific inhibitor reduces amyloid beta-mediated brain disorder in a mouse model of Alzheimer disease. J Clin Invest 122: 1377-1392, 2012

7. Erickson MA, Hartvigson PE, Morofuji Y, Owen JB, Butterfield DA and Banks WA: Lipopolysaccharide impairs amyloid $\beta$ efflux from brain: Altered vascular sequestration, cerebrospinal fluid reabsorption, peripheral clearance and transporter function at the blood-brain barrier. J Neuroinflammation 9: 150, 2012.

8. Ko SY, Ko HA, Chu KH, Shieh TM, Chi TC, Chen HI, Chang WC and Chang SS: The possible mechanism of advanced glycation end products (AGEs) for Alzheimer's disease. PLoS One 10: e0143345, 2015.

9. Wang X, Yu S, Hu JP, Wang CY, Wang Y, Liu HX and Liu YL: Streptozotocin-induced diabetes increases amyloid plaque deposition in $\mathrm{AD}$ transgenic mice through modulating AGEs/RAGE/NF-kB pathway. Int J Neurosci 124: 601-608, 2014.

10. Lv C, Wang L, Liu X, Yan S, Yan SS, Wang Y and Zhang W: Multi-faced neuroprotective effects of geniposide depending on the RAGE-mediated signaling in an Alzheimer mouse model. Neuropharmacology 89: 175-184, 2015.

11. Di BB, Li HW, Li WP, Shen XH, Sun ZJ and Wu X: Pioglitazone inhibits high glucose-induced expression of receptor for advanced glycation end products in coronary artery smooth muscle cells. Mol Med Rep 11: 2601-2607, 2015.

12. Xiong MQ (ed): Theory on Exogenous Febrile Disease. China Press of Traditional Chinese Medicine, Beijing, 2007.

13. Yu B, Zhou C, Zhang J, Ling Y, Hu Q, Wang Y and Bai K: Latest study on the relationship between pathological process of inflammatory injury and the syndrome of spleen deficiency and fluid retention in Alzheimer's disease. Evid Based Complement Alternat Med 2014: 743541, 2014.

14. Sang F: Experimental research on the mechanism of Alzheimer's disease. J Tradit Chin Med 6: 685-687, 2011.

15. National Research Council: Guide for the Care and Use of Laboratory Animals: Eighth Edition. Guide for the Care \& Use of Laboratory Animals. The National Academies Press, pp1072-1073, 2010.
16. Maurice T, Lockhart BP and Privat A: Amnesia induced in mice by centrally administered beta-amyloid peptides involves cholinergic dysfunction. Brain Res 706: 181-193, 1996.

17. Cheng YF, Wang C, Lin HB, Li YF, Huang Y, Xu JP and Zhang HT: Inhibition of phosphodiesterase- 4 reverses memory deficits produced by $A \beta 25-35$ or $A \beta 1-40$ peptide in rats. Psychopharmacology (Berl) 212: 181-191, 2010.

18. Kwon SH, Lee HK, Kim JA, Hong SI, Kim SY, Jo TH, Park YI, Lee CK, Kim YB, Lee SY and Jang CG: Neuroprotective effects of Eucommia ulmoides, Oliv. Bark on amyloid beta (25-35)-induced learning and memory impairments in mice. Neurosci Lett 487: 123-127, 2011.

19. Klunk WE, Jacob RF and Mason RP: Quantifying amyloid beta-peptide (Abeta) aggregation using the Congo red-Abeta (CR-abeta) spectrophotometric assay. Anal Biochem 266: 66-76, 1999.

20. Eisele YS, Obermüller U, Heilbronner G, Baumann F, Kaeser SA, Wolburg H, Walker LC, Staufenbiel M, Heikenwalder M and Jucker M: Peripherally applied Abeta-containing inoculates induce cerebral beta-amyloidosis. Science 330: 980-982, 2010.

21. Livak KJ and Schmittgen TD: Analysis of relative gene expression data using real-time quantitative PCR and the 2(-Delta Delta C(T)) method. Methods 25: 402-408, 2001

22. Golden HL, Agustus JL, Nicholas JM, Schott JM, Crutch SJ, Mancini L and Warren JD: Functional neuroanatomy of spatial sound processing in Alzheimer's disease. Neurobiol Aging 39: 154-164, 2016.

23. Yu B: Study on the relationship between Alzheimer's disease and the syndrome of spleen deficiency and fluid retention based on Lingguizhugantang's intervention of $\mathrm{A} \beta$-induced inflammatory injury (unpublished $\mathrm{PhD}$ dissertation). Nanjing University of TCM, 2015

24. Harrington KD, Lim YY, Gould E and Maruff P: Amyloid-beta and depression in healthy older adults: A systematic review. Aust N Z J Psychiatry 49: 36-46, 2015.

25. Jeynes B and Provias J: Evidence for altered LRP/RAGE expression in Alzheimer lesion pathogenesis. Curr Alzheimer Res 5: 432-437, 2008.

26. Deane R, Bell RD, Sagare A and Zlokovic BV: Clearance of amyloid-beta peptide across the blood-brain barrier: Implication for therapies in Alzheimer's disease. CNS Neurol Disord Drug Targets 8: 16-30, 2009.

27. Christensen DD: Higher-dose ( $23 \mathrm{mg} /$ dayay) donepezil formulation for the treatment of patients with moderate-to-severe Alzheimer's disease. Postgrad Med 124: 110-116, 2012.

28. Nordberg A: Mechanisms behind the neuroprotective actions of cholinesterase inhibitors in Alzheimer disease. Alzheimer Dis Assoc Disord 20 (2 Suppl 1): S12-S18, 2006.

29. Rogers SL, Farlow MR, Doody RS, Mohs R and Friedhoff LT: A 24-week, double-blind, placebo-controlled trial of donepezil in patients with Alzheimer's disease. Donepezil Study Group. Neurology 50: 136-145, 1998.

30. Agboton C, Mahdavian S, Singh A, Ghazvini P, Hill A and Sweet A: Impact of nighttime donepezil administration on sleep in the older adult population: A retrospective study. Mental Health Clinician 4: 257-259, 2014.

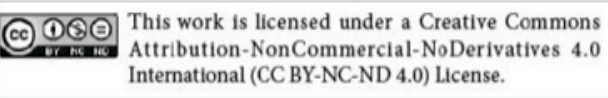

